\title{
SCALING OF ENERGY GAIN WITH PLASMA PARAMETERS IN A PLASMA WAKEFIELD ACCELERATOR
}

\author{
P. Muggli, T. Katsouleas, E. Oz, USC, Los Angeles CA, USA
}

\author{
I. Blumenfeld, F.-J. Decker, M.J. Hogan, R. Ischebeck, R. Iverson, N. Kirby, R. Siemann, D. Walz, \\ SLAC, Menlo Park, USA
}

\author{
C.E. Clayton, C. Huang, C. Joshi, W. Lu, K.A. Marsh, W.B. Mori, M. Zhou, UCLA, Los Angeles, \\ USA
}

\begin{abstract}
We have recently demonstrating the doubling of the energy of particles of the ultra-short, ultra-relativistic electron bunches of the Stanford Linear Accelerator Center [1]. This energy doubling occurred in a plasma only $85 \mathrm{~cm}$-long with a density of $\approx 2.6 \times 10^{17} \mathrm{e}^{-} / \mathrm{cm}^{-3}$. This milestone is the result of systematic measurements that show the scaling of the energy gain with plasma length and density, and show the reproducibility and the stability of the acceleration process. We show that the energy gain increases linearly with plasma length from 13 to $31 \mathrm{~cm}$. These are key steps toward the application of beamdriven plasma accelerators or plasma wakefield accelerators (PWFA) to doubling the energy of a future linear collider without doubling its length.
\end{abstract}

\section{INTRODUCTION}

Plasma accelerators have two great potential advantages when compared to conventional, radiofrequency accelerators. First, since the accelerating structure is sustained by the plasma itself, there are no fabrication issues related to operation at high frequencies (for example $2.8 \mathrm{THz}$ or a wavelength of $106 \mu \mathrm{m}$ at a plasma density of $10^{16} \mathrm{~cm}^{-3}$ ). Second, plasmas can sustain plasma waves or wakes with extremely large accelerating gradients, in excess of $10 \mathrm{GV} / \mathrm{m}$. It is therefore important to explore the possibility of using plasmas to accelerate particles to multi-GeV energies. In this context, we are studying a particle beam-driven plasma accelerator known as the plasma wakefield accelerator or PWFA.

We have demonstrated that large accelerating gradients can be excited in plasma $\mathrm{s}$ by short electron $\left(\mathrm{e}^{-}\right)$and positron $\left(\mathrm{e}^{+}\right)$bunches $[1,2,3,4]$. To lead to a large energy gain, these gradients need to be sustained over long plasma lengths. We have set up a PWFA experiment with a variable plasma length in order to study the scaling of energy gain with plasma length and density, and to demonstrate that the energy gain increases linearly with the plasma length.

\section{THE PLASMA WAKEFIELD ACCELERATOR}

The PWFA is driven by the ultra-short $\left(\sigma_{z}>10 \mu \mathrm{m}\right) \mathrm{e}^{-}$ bunches of the Stanford Linear Accelerator Center
(SLAC). Individual bunches with $\approx 1.8 \times 10^{10}, 28.5 \mathrm{GeV} \mathrm{e}^{-}$ are focused to a transverse size in vacuum $\sigma_{r}$ of $\approx 10 \mu \mathrm{m}$ near the entrance of a lithium $(\mathrm{Li})$ vapor column located near the focal point of the SLAC FFTB line. A schematic of the experimental setup is shown on Fig.1. The Li vapor is produced in a heat-pipe oven [5]. The oven consists of a $3.8 \mathrm{~cm}$ diameter stainless steel tube containing a fine stainless steel mesh wick and surrounded by high temperature heaters. The oven is insulated from the beam line high vacuum by $25 \mu \mathrm{m}$ thick beryllium windows located at each end. The oven is initially filled with a helium (He) buffer gas at a given pressure and solid $\mathrm{Li}$ is placed in the heaters region. The oven is heated until the Li melts and its vapor temperature is equal to that of the buffer gas. In that "oven mode" the room temperature $\mathrm{He}$ confines the pure, hot Li column to the hot zone of the oven [6]. The Li density is determined by the buffer gas pressure, and the Li column length by the length of the heater and is adjustable in three lengths $L_{p}=13,22,31 \mathrm{~cm}$ while the location of the column entrance is kept fixed. The length of the Li column is calculated from the longitudinal temperature profile of the vapor obtained by pulling a thermocouple probe through the oven. Lithium was chosen because of the low ionization potential of its first $\mathrm{e}^{-}(5.392 \mathrm{eV})$ and the relatively high potential for its two subsequent $\mathrm{e}^{-}(75.638$ and $122.455 \mathrm{eV})$. In previous long bunch $\left(\sigma_{\mathrm{z}} \approx 700 \mu \mathrm{m}\right)$ PWFA experiments the plasma was created through photo-ionization of the $\mathrm{Li}$ vapor by an ultra-violet laser pulse [2, 5]. However, in the present experiments the transverse electric field of the ultra-short $\mathrm{e}^{\mathrm{e}}$ bunches is large enough to field-ionize the first $\mathrm{Li} \mathrm{e}$ over a time scale shorter than the bunch duration [7]. The ADK theory for field ionization [8] indicated that full ionization occurs in the volume surrounding the bunch where the electric field exceeds $\approx 6 \mathrm{GV} / \mathrm{m}$. With the SLAC bunches full ionization extends over a radius of more than $100 \mu \mathrm{m}$ and starts much before the peak of the bunch current. Ionization over a radius larger than the plasma collisionless skin depth $\left(c / \omega_{p}\right.$, where $\omega_{p}=\left(n_{e} e / \varepsilon_{0} m_{e}\right)^{1 / 2}$ is the plasma angular frequency) is necessary for the wake to be similar to that in a preformed plasma. Once Li is ionized, the bunch fields expel the plasma $\mathrm{e}^{-}$from the bunch volume and lose energy to the plasma wake. When the beam density $n_{b}$ is larger than 
the plasma density $n_{e}$, all the plasma electrons are expelled from the bunch volume and a pure ion column is left behind the head of the bunch. The ion column partially $\left(n_{i}=n_{e}<n_{b}\right)$ neutralizes the relativistic $\mathrm{e}^{-}$bunch, which is in turn focused. In this regime, the focusing field of the ion column is given by $E_{r}(r)=(1 / 2)\left(n_{e} e / \varepsilon_{0}\right) r$ leading to an aberration-free focusing for the core of the bunch. The ion column also acts as restoring force for the plasma $\mathrm{e}^{-}$, which rush back on axis about one plasma wavelength after being expelled. They create an on-axis negative density spike, which then accelerates the $\mathrm{e}^{-}$in the back of the bunch. The linear theory for the single bunch PWFA [9] indicates that, for a bunch with a small radius compared to $c / \omega_{p}$, the largest accelerating gradient is achieved when the bunch length and plasma density are such that $k_{p} \sigma_{z}=\left(\omega_{p} / c\right) \sigma_{z} \approx \sqrt{ } 2$, and scales as $E_{\text {acc. } \max } \approx 110 M V\left(N / 2 \times 10^{10}\right) /\left(\sigma_{z}(\mu m) / 700\right)^{2}$. Note that in a single bunch experiment the particle bunch fills all the phases of the accelerating bucket, and a continuum of energy loss and gain is observed. In a real accelerator configuration, the plasma wake is driven by a high charge bunch that only loses energy, while a short $\left(<<2 \pi / k_{p}\right)$, lower charge witness bunch is accelerated with a narrow energy spread. The plasma accelerator therefore provides both focusing and acceleration for the witness bunch, and the energy is extracted from the drive bunch through the plasma wake.

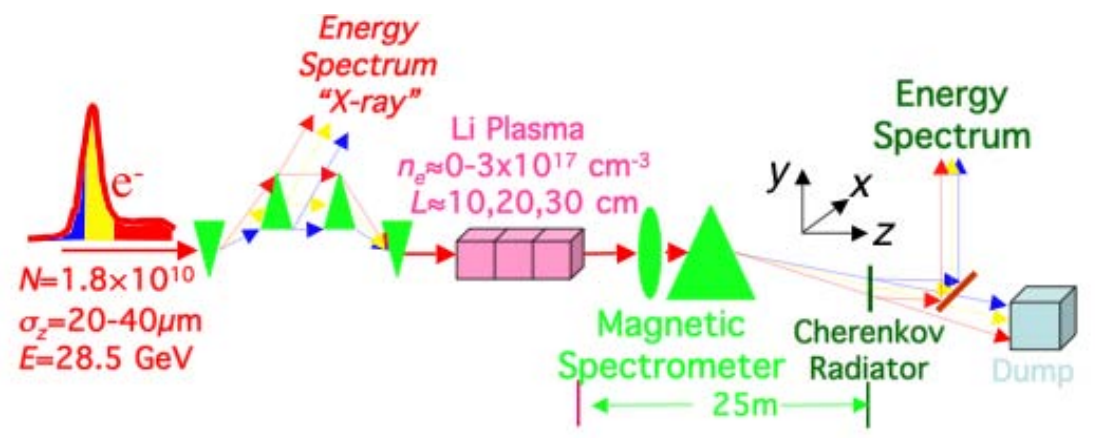

Figure 1: Schematic of the experimental setup (not to scale). The bunch incoming energy spectrum (before the plasma) is measured from the synchrotron radiation emitted by $\mathrm{e}^{-}$in a weak vertical magnetic chicane placed in a region of the FFTB line with horizontal dispersion. After the plasma the beam travels through a magnetic spectrometer consisting of quadrupole and dipole magnets. The energy spectrum is measured again using Cherenkov radiation emitted by particles in a thin piece of aerogel.

\section{EXPERIMENTAL RESULTS}

In the experiment, the bunch interacts with the plasma and is then transported through an magnetic spectrometer consisting of a combination of quadrupole and dipole magnets. The spectrometer is designed to ensure that in the $y$-dispersion direction of the energy measurement plane the beam size $\sigma_{y}$ is dominated by dispersion and not by the natural beam size associated with its emittance $\varepsilon_{y}$ $\sigma_{y}=\left(\beta_{y} \varepsilon_{y}+\left(\eta \Delta E / E_{0}\right)^{2}\right)^{1 / 2} \approx \eta \Delta E / E_{0}, \quad$ where $\quad \beta_{y}=\sigma_{y \varepsilon}{ }^{2} / \varepsilon_{y}$ is the beam beta function in the $y$-dispersive plane $\Delta E$ is the bunch energy content around the incoming energy $E_{0}$, and $\eta$ is the dispersion in the energy diagnostic plane. This spectrometer removes the ambiguity between energy gain and the transverse momentum that can be imparted by the strong plasma focusing force to a possible off-axis beam tail at the plasma entrance. The bunch energy spectrum is also measured before the plasma using the synchrotron radiation emitted by the $\mathrm{e}^{-}$in a weak vertical magnetic chicane placed in a dispersive region of the FFTB. This spectrum allows for the identification of bunches with similar incoming longitudinal phase space $\left(z-p_{z}\right)$ [4]. It is also used to retrieve the bunch current profile by comparison with simulation results of the bunch compression process using the code LiTrack [10].
Simulation results indicate that the bunches used in this experiment have a low current $(<2 \mathrm{kA})$ "trunk" followed by a bi-Gaussian peak with a current in the 6-16 kA (for $\approx 1.8 \times 10^{10} \mathrm{e}^{-/}$bunch) with an average rms width between 20 and $40 \mu \mathrm{m}$.

Energy spectra measured after plasmas with a density of $n_{e}=2.7 \times 10^{17} \mathrm{~cm}^{-3}$ and lengths $L_{p}=13 \mathrm{~cm}, 22 \mathrm{~cm}$, and $31 \mathrm{~cm}$ obtained with bunches with similar incoming parameters show that the energy gain increases linearly with the plasma length. The maximum energy gain is $\approx 41 \mathrm{GeV}$ after the $31 \mathrm{~cm}$ long plasma, or an energy gain of about $12.5 \mathrm{GeV}$. This energy gain corresponds to an average accelerating gradient of the order of $\approx 40 \mathrm{MV} / \mathrm{m}$ that is driven and sustained along the $31 \mathrm{~cm}$ long plasma. In these experiments, the energy gain is limited by the plasma length and by the amount of charge that can be lost along the beam line section located after the plasma. Measurements with longer bunch and lower plasma densities indicate that the measurable energy loss is limited by interception of the low energy particles $(<24 \mathrm{GeV})$ along the beam line and on protection collimators. The energy loss is expected to be of the same order as the peak energy gain. Systematic measurements indicate that the energy gain scales linearly with the three plasma lengths available in this experiment $\left(L_{p}=13,22\right.$, $31 \mathrm{~cm})$. This dependency is also observed at plasma 
densities that lead to over all lower energy gains [11]. These results suggest that the energy of $28.5 \mathrm{GeV} \mathrm{e}^{-}$can be doubled in a $n_{e}=2.7 \times 10^{17} \mathrm{~cm}^{-3}$, only about $70 \mathrm{~cm}$ long plasma. The successful energy doubling experiment was performed with $42 \mathrm{GeV}$ bunches to maximize the absolute energy gain [1]. The energy spectrometer was also modified to account for the very broad energy spectrum of the beam after interaction with the plasma [12].

\section{IMPLICATIONS FOR AN ENERGY DOUBLER}

The results presented in this paper and previously published [1] represent significant steps toward the possible realization of an energy doubler for a future $\mathrm{e}^{-} / \mathrm{e}^{+}$ collider, an afterburner scheme proposed [13]: the energy gain by $\mathrm{e}^{-}$scales with plasma length and leads to energy doubling over only $85 \mathrm{~cm}$. The unloaded accelerating gradient is in excess of $40 \mathrm{GV} / \mathrm{m}$ and sustained over long plasma lengths. This gradient is larger than the 10$20 \mathrm{GeV}$ value envisaged for the afterburner. In that scheme the plasma length for energy doubling will be in the tens of meters range to reach 0.5 to $1 \mathrm{TeV}$.

With the extremely small beam sizes and emittances needed to reach the desired collision luminosity, new issues may be a challenge for the plasma afterburner. For example erosion of the head of the drive bunch would lead to evolution of the wake amplitude and shape along the plasma [14]. Motion of the plasma ions induced by the strong field of the drive bunch could lead to degradation of the emittance of the witness bunch $[15,16]$. Coupling between transverse motion of the plasma $\mathrm{e}^{-}$forming the accelerating structure and the bunch $\mathrm{e}^{-}$can seed the growth of the well known hose instability which could limit the energy gain, produce emittance growth or even destroy the bunches. All these effects will have to be studied numerically and experimentally. The beam and plasma parameters will have to be chosen carefully to mitigate the deleterious effect associated with these issues. Future experiments will focus on the acceleration of a witness $\mathrm{e}^{-}$bunch to a narrow energy spectrum and detailed studies of positron/plasma interaction at the new SLAC beam facility [17].

\section{ACKNOWLEDGEMENTS}

The authors would like to thank Dr. Peter Tsou of JPL for the aerogel. Work supported by US DoE under contracts DE-AC02-76SF00515, DE-FG03-92ER40745, DE-FG03-98DP00211, DE-FG03-92ER40727, DE-AC0376SF0098 and NSF Grants ECS-9632735, DMS9722121, PHY-0078715.

\section{REFERENCES}

[1] I. Blumenfeld et al., Nature 445, 741-744 (15 February 2007).

[2] P. Muggli et al., Phys. Rev. Lett. 93, 014802 (2004).
[3] B.E. Blue et al., Phys. Rev. Lett. 90, 214801 (2003).

[4] M. J. Hogan et al., Phys. Rev. Lett. 95, 054802 (2005).

[5] P. Muggli et al., IEEE Trans. on Plasma Science 27(3), pp. 791-799 (1999).

[6] C. R. Vidal and J. Cooper, J. Appl. Phys., 40, 3370 (1969).

[7] C.L. O'Connell et al., Phys. Rev. ST-AB 9, 101301 (2006).

[8] M. V. Ammosov, N. B. Delone, and V. P. Krainov, Sov. Phys. JETP 64, 1191 (1986).

[9] S. Lee et al., Phys. Rev. E 61, 7014 (2000).

[10] K. L. F. Bane et al., Stanford Linear Accelerator Center Report No. SLAC-PUB-11035, 2005 (unpublished).

[11] P. Muggli et al., to be submitted.

[12] R. Ischebeck et al., these proceedings.

[13] S. Lee et al., Phys. Rev. ST Accel. Beams 5, 121301 (2002).

[14] C. Huang et al., Proceedings of the 2005 Particle Accelerator Conference, Knoxville, Tennessee, 2666, (2005).

[15] J.B. Rosenzweig et al., Phys. Rev. Lett. 95, 195002 (2005).

[16] R. Gholizadeh et al., these proceedings.

[17] R. Erickson et al., these proceedings. 